\title{
Persistence and plant uptake of methylarsenic in continuously- and intermittently-flooded rice paddies
}

\author{
S.C. Maguffin ${ }^{1,2}$, M.C. Reid ${ }^{1,2}$, A. McClung ${ }^{1,2}$ \& J. Rohila ${ }^{1,2}$ \\ ${ }^{1}$ Civil \& Environmental Engineering, Cornell University, Ithaca, NY, USA \\ ${ }^{2}$ Dale Bumpers National Rice Research Center, Stuttgart, AR, USA
}

\begin{abstract}
Rice grown in the South-Central USA tends to accumulate a larger fraction of methylarsenic species than rice grown in other regions, and the reason for these patterns are not clear. The goal of this study was to determine how soil management conditions influence the persistence and plant uptake of methylarsenic species in rice paddy soils, using field-scale experimental plots in Arkansas, USA. We expect methylated arsenic to persist in pore water and be more abundant in biomass in continuously flooded grown rice.
\end{abstract}

\section{INTRODUCTION}

Rice accumulates arsenic (As) primarily as inorganic arsenic and the methylarsenic species dimethylarsinic acid (DMAs(V)) (Ma et al., 2008). Rice grown in the South-Central United States tends to accumulate a larger fraction of $\mathrm{DMAs}(\mathrm{V})$ than rice grown in other regions. The reasons for these patterns remain unclear, and these uncertainties underscore a broader lack of knowledge around how the persistence and plant availability of methylarsenic species in rice paddy soils are governed by soil environmental conditions (Zhao et al., 2013).

The goal of this study was to determine the persistence and fate of As originating from the methylarsenic-based pesticide monosodium methanearsonate (MSMA) in field-scale experimental rice plots cultivated under different soil management conditions. The specific objectives of this study were: (1) Monitor MSMA in pore waters and associated with soil minerals to determine the effect of irrigation regime on plant-available methylarsenic species; and (2) Track As abundance and speciation in rice plant tissues at different stages of maturity to examine links between belowground As dynamics and As accumulation in plant tissues, with a special focus on rice grains.

\section{METHODS AND EXPERIMENTAL DESIGN}

\subsection{Experimental design}

A field monitoring study was conducted during the 2017 growing season at the United States Department of Agriculture Dale Bumpers National Rice Research Center in Stuttgart, Arkansas, USA, as part of a multidecade study on the impacts of MSMA amendment on As uptake into rice. A $2 \times 2$ factorial experimental design (Fig. 1) was used to test the effects of irrigation regime (continuously-flooded (CF) vs. alternate

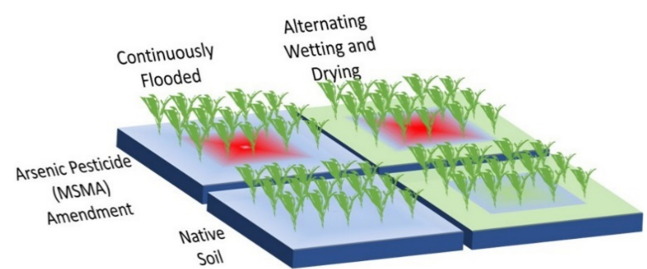

Figure 1. Conceptual illustration of the $2 \times 2$ factorial experimental design at USDA-ARS Dale Bumpers Research Service. The red represents the application of the MSMA pesticide onto the rice paddy.

wetting and drying (AWD)) and MSMA amendment (1.1 mg-As kg ${ }^{-1}$ - applied annually for more than 20 years) on As speciation in pore water as well as As associated with soil solid phases.

\subsection{Soil solution sampling}

Three pore water sampling locations were established in each experimental field. Each location had a Rhizon pore water sampler at $10 \mathrm{~cm}$ and $25 \mathrm{~cm}$ depth. Pore water samplers were equipped with $0.15 \mu \mathrm{M}$ filters and were anaerobically sampled bi-weekly starting 3 weeks after plots were planted using a vacuumed, autoclaved, acid-washed bottle. In the MSMA-amended AWD experiment, we sampled pore water at a higher frequency during a mid-season wetting event to more closely examine the effects of soil wetting and drying on aqueous chemistry and arsenic speciation.

Pore water arsenic speciation was determined using HG-CT-GC-AAS. Pore water samples were also analyzed for inorganic anions using ion chromatography, total elemental analysis using ICP-OES, and dissolved organic carbon (DOC) using a total organic carbon analyzer. Soil temperature moisture were logged using in situ probes. 


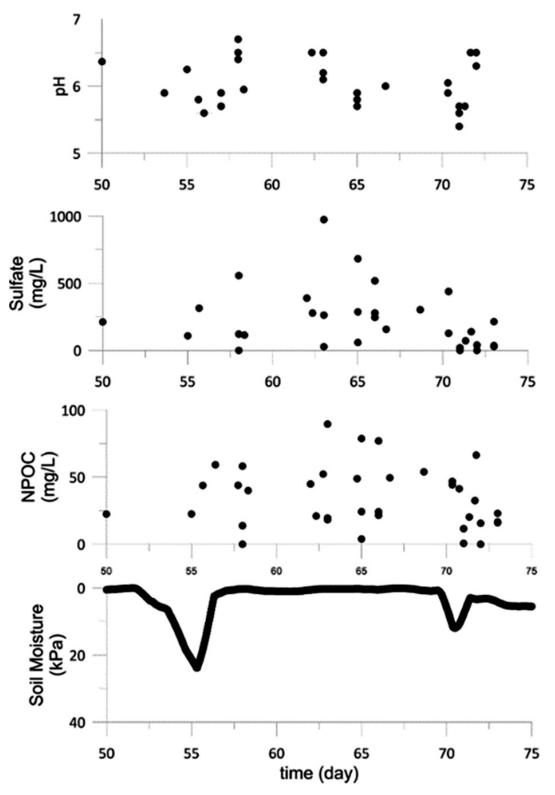

Figure 2. Mid-growing season high resolution aqueous sampling data from the AWD-MSMA-amended field's shallow wells. Alongside regular sampling, high-frequency sampling was conducted to more clearly resolve arsenic redox and speciation chemistry during specific alternating wetting and drying events.

\subsection{Solid phase analysis}

Soil was collected using a soil probe and anaerobically preserved in heat sealed BoPET bags flushed with research grade argon gas. Total elemental analysis was performed via XRF and by acid digestion followed by ICP-OES. As speciation was determined using X-ray absorption near-edge structure (XANES) at the Cornell High Energy Synchrotron Source (CHESS).

\section{EXPECTED RESULTS AND DISCUSSION}

\subsection{Pore water}

Initial results indicate a difference in pore water chemistry between irrigation methods. Soil solution chemistry was more greatly affected by AWD controls at $10 \mathrm{~cm}$ depth than at $25 \mathrm{~cm}$. In the MSMA-AWD experiment, high resolution sampling highlighted the effect soil moisture has on $\mathrm{pH}$, sulfate, and DOC (Fig. 2). An increase in soil moisture correlated strongly with an increase in sulfate and DOC before sulfate reducing conditions were reached and both decreased (Fig. 2). Pore water As analyses have not been completed at the time of abstract submission.

\subsection{Solid phase}

XANES analysis was employed to speciate the arsenic within soil at the beginning and end of the growing season (Fig. 3). Importantly, arsenic in soil from the MSMA-flooded experiment collected at the end of the

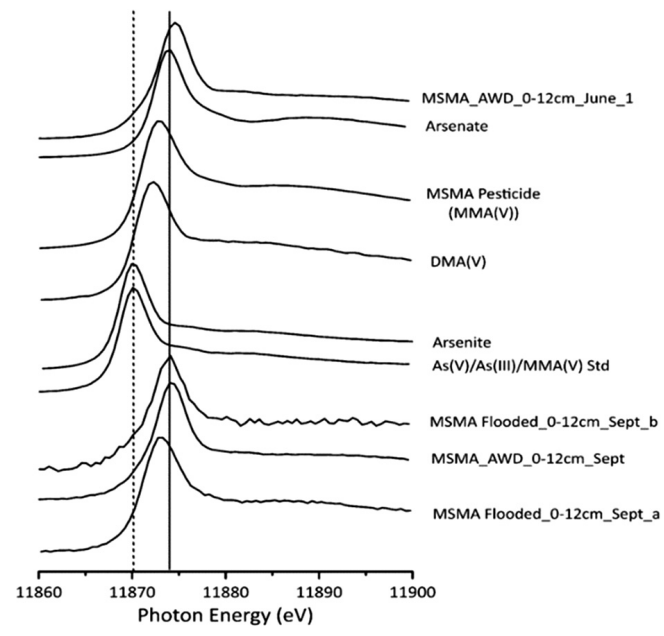

Figure 3. XANES spectra of reference standards and soil samples from flooded and AWD fields. The solid vertical line indicates the arsenate white line energy. The dotted vertical line indicates the arsenite white line energy (not all data shown).

growing season was different than arsenate's white line energy; this indicates that MSMA was not completely demethylated to arsenite and/or arsenite was not completely oxidized to arsenate. This was not the case for results from MSMA-AWD experiments and thus suggests a persistence of MSMA in flooded paddy soils is possible.

\section{CONCLUSIONS}

Data from arsenic analyses, combined with pore water and solid phase chemistry, will provide a detailed characterization of methylation chemistry in rice paddy soils as a function of irrigation controls and background arsenic concentrations. Expected results will highlight differences in arsenic speciation among experimental designs.

\section{ACKNOWLEDGEMENTS}

This work has been funded through the National Science Foundation Postdoctoral Fellowship Program and Cornell University.

\section{REFERENCES}

Ma, J.F., Yamaji, N., Mitani, N., Xu, X.-Y., Su, Y.-H., McGrath, S. P. \& Zhao, F.-J. 2008. Transporters of arsenite in rice and their role in arsenic accumulation in rice grain. Proc. Natl. Acad. Sci. U.S. A. 105(29): 9931-9935.

Zhao, F.J., Zhu, Y.G., \& Meharg, A.A. 2013. Methylated arsenic species in rice: geographical variation, origin, and uptake mechanisms. Environ. Sci. Technol. 47(9): 3957-3966. 